\title{
Procedimento de segurança para manuseio de nanomateriais em laboratórios
}

\section{Safety procedure for handling nanomaterials in laboratories}

DOI: $10.54021 /$ seesv1n1-001

\author{
Samuel Jônatas de Castro Lopes \\ Engenheiro Civil e de Segurança no Trabalho \\ Especialista em Engenharia de Avaliações e Perícias \\ Mestre em Ciência e Engenharia de Materiais \\ Doutorando em Ciência dos Materiais
}

\section{RESUMO}

Introdução: a nanotecnologia vem propiciando uma revolução tecnológica na engenharia mundial. Dentre os nanomateriais mais utilizados, os nanotubos de carbono (NTC) se destacam por suas diversas propriedades excelentes e específicas. Entretanto, as implicações a saúde de usuários que manipulam os nanotubos de carbono estão longe de serem compreendidas e cercadas de incertezas. Métodos: estudo exploratório-descritivo, baseado em uma breve contextualização acerca dos nanotubos de carbono e da nanotoxicologia, em busca de compreender as principais características morfológicas deste material, e os principais danos causados à saúde dos trabalhadores. Objetivo: propor um procedimento de segurança para manuseio de nanomateriais em laboratórios. Resultados: os laboratórios que manipulam esse tipo de tecnologia devem ser dotados de instalações específicas e possuir procedimentos operacionais que busquem controlar a exposição e a contaminação aerotransportada. Conclusão: a compatibilização da cadeia comercial e a segurança do trabalho devem ser consideradas para nanomateriais, ou seja, o diálogo entre fabricante, fornecedor, usuário final e profissionais de saúde e segurança do trabalho (SST) é de suma importância para o desenvolvimento da nanotecnologia segura.

Palavras-chaves: nanotecnologia; procedimento de segurança; laboratório.

\section{ABSTRACT}

Introduction: nanotechnology has led to a technological revolution in world engineering. Among the most used nanomaterials, carbon nanotubes (CNT) stand out for their excellent and specific properties. However, the health impacts of users who manipulate carbon nanotubes are far from being understood and surrounded by uncertainties. Methods: exploratory-descriptive study based on a brief contextualization about carbon nanotubes and nanotoxicology, in an attempt to understand the main morphological characteristics of this material, and the main damages caused to workers' health. Objective: a safety procedure for handling nanomaterials in laboratories was proposed. Results: Laboratories that handle this type of technology must be equipped with specific facilities and have operational 
procedures that seek to control exposure and airborne contamination. Conclusion: the compatibility of the commercial chain and work safety must be considered for nanomaterials, that is, the dialogue between manufacturer, supplier, end user and and occupational health and safety professionals is of paramount importance for the development of safe nanotechnology.

Keywords: nanotechnology; safety procedure; laboratory.

\section{INTRODUÇÃO}

A nanotecnologia vem propiciando uma revolução tecnológica na engenharia mundial; isso se deve a sua capacidade em manipular a matéria a nível atômico formando estruturas estáveis que estão compreendidas entre 1 e 100 nanômetros (nm) [1]. Essa tecnologia tem diversas aplicações na medicina e agricultura, uma vez que esses setores utilizam conhecimentos específicos de física, química, ciência dos materiais, ciência da computação e eletrônica [2].

Dentre os nanomateriais mais utilizados, os nanotubos de carbono (NTC) se destacam por suas diversas propriedades excelentes e específicas, tais como: alta condutividade térmica e elétrica, alta resistência à tração e baixo peso específico [3]. Entretanto, as implicações a saúde de usuários que manipulam os nanotubos de carbono estão longe de serem compreendidas e cercadas de incertezas [4]. Devido seu baixo peso específico, os nanotubos de carbono, podem permanecer no ambiente de trabalho como material particulado em suspensão, representando um alto risco por conta da possibilidade de inalação ${ }^{5}$. É sabido afirmar que altas exposições podem causar danos à saúde dos pesquisadores que manuseiam esses nanomateriais em laboratórios, caso o ambiente não esteja devidamente controlado. Além disso, estudos acerca da toxicidade dos NTC ainda são bastante complexos, devido a singularidade de cada nanopartícula, gerando avaliações contraditórias e colocando em risco, teoricamente, a saúde das pessoas que manipulam este material [5]. As contradições encontradas com relação à toxicidade dos nanotubos de carbono são devidas especialmente a diversidade entre eles, uma vez que, cada NTC apresenta características diferentes tanto quanto a seu uso como quanto a toxicidade.

Os atuais métodos quantitativos utilizados pela higiene ocupacional $(\mathrm{HO})$ não contemplam especificamente nanomateriais, portanto, existe uma fragilidade no gerenciamento de riscos nestes ambientes de trabalho [6]. Atualmente, vem 
sendo utilizada a técnica qualitativa de controle por faixas com objetivo de realizar a avaliação de risco à exposição aos nanomateriais de maneira simplificada [7]. É de extrema relevância discutir os possíveis efeitos negativos a saúde dos trabalhadores, assim como propor um procedimento para mitigar os potenciais riscos existentes no ambiente de trabalho [8]. Motivado por isso, o presente estudo apresenta uma breve contextualização acerca dos nanotubos de carbono e da nanotoxicologia, em busca de compreender as principais características morfológicas deste material, e os principais danos causados à saúde dos trabalhadores. Posteriormente, foi proposto um procedimento de segurança para manuseio de nanomateriais em laboratórios.

\section{NANOTUBOS DE CARBONO}

Os fulerenos são moléculas que possuem 60 átomos de carbono (C) na sua estrutura e se caracterizam por ser uma forma alotrópica de C [9]. Os nanotubos de carbono pertencem a família dos fulerenos e foram descobertos 1991 por lijima [10]. Os NTC são comumente tratados como materiais unidimensionais, pois sua razão comprimento-diâmetro gira em torno de 1000 unidades arbitrárias [11]. Diversas rotas de síntese são utilizadas para obtenção deste nanomaterial, tais como: descarga de arco, eletrólise e deposição química a vapor (CVD, na siga em inglês) [12] [13] [14]. O método de síntese CVD é ideal para obtenção de nanotubos de carbono, devido à simplicidade e aos mecanismos de controle da orientação, comprimento e diâmetro [15]. Os NTC são materiais extremamente fortes e flexíveis, possuindo 100 vezes a resistência e 6 vezes mais a leveza do aço [11]. Na figura 1, é possível observar a ilustração de um NTC.

Esses materiais podem ser classificados segundo o seu arranjo cilíndrico, resultando em duas categorias: nanotubos de carbono de parede simples (NCPS) e nanotubos de carbono de paredes múltiplas (NCPM). O primeiro tipo é formado apenas por uma folha de grafeno, em forma cilíndrica, com diâmetros entre 0,4 e 2,0 nm [16]. Por outro lado, os NCPM são compostos por vários NCPS concêntricos como em um cabo coaxial [17]. Os nanotubos de carbono de paredes múltiplas possuem um diâmetro que varia entre 2,0 e $100 \mathrm{~nm}$, sendo este parâmetro influenciado pelo número de camadas [16]. 
Figura 1 - Representação esquemática de um nanotubo de carbono com parede simples

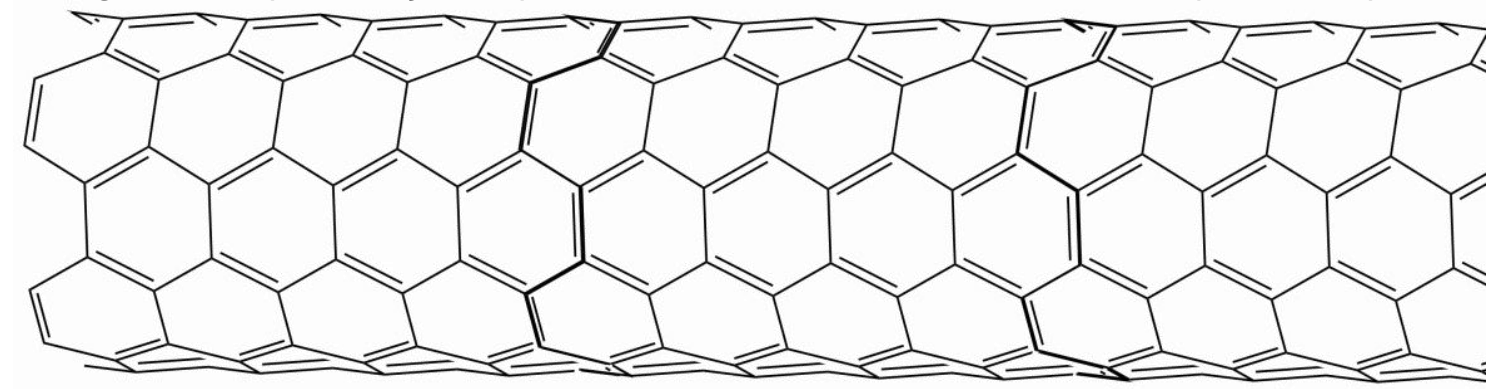

Fonte: Modificada a partir de [11]

Outra forma de categorizar os NTCs é quanto à sua capacidade de aglomeração. Esse modelo de classificação é de suma importância para o campo da medicina, sendo dividido em dois principais tipos: estruturas com tendência de se aglomerar (I) e dispersar (II). Os aglomerados de NTC ficam suspensos no ar e tendem a se depositar nos alvéolos proximais, através da inalação, podendo causar granulomas. Por outro lado, os NTC com tendência a se dispersar se depositam nos alvéolos distais, podendo causar fibrose intersticial [18] [19] [20].

\section{NANOTOXICOLOGIA}

O fato de os nanotubos de carbono possuírem propriedades inéditas, proporcionando uma gama de aplicações, também os torna acessíveis a locais anteriormente inacessíveis em seres humanos, podendo gerar consequências graves a saúde [21]. Partículas grossas, tais como poeiras carregadas pelo vento possuem diâmetro que variam entre $2 \times 10^{3}$ e $1 \times 10^{5}$ nanômetros. Por outro lado, as partículas finas possuem diâmetro compreendidos no intervalo entre 1 e 2000 nanômetros [22].

Os nanomateriais são caracterizados por suas pequenas dimensões e baixo peso específico, podendo sua dispersão no ambiente de trabalho ocasionar potenciais riscos à saúde dos pesquisadores [4] [23]. Desta forma, os NTC podem ser absorvidos através do processo de inalação, atingindo os alvéolos pulmonares. É sabido afirmar que a absorção pelo trato respiratório não é a única rota de exposição para riscos biológicos, podendo a ingestão, penetração na pele e mucosa serem classificadas como rotas alternativas [24]. A figura 2 demonstra a relação entre as diferentes regiões do sistema respiratório e a fração inalada de partículas, localizada no eixo $\mathrm{x}$ e y, respectivamente. Três regiões do trato respiratório são representadas na figura 2: nasofaringe, traqueobrônquica (T-B) e 
pulmonar. Partículas maiores, representadas por triângulos azuis, se depositam preferencialmente na região da nasofaringe, enquanto partículas menores, caracterizadas por quadrados pretos, tendem a se aglomerar em maior quantidade na região pulmonar. Essas pequenas partículas podem ocasionar estresse oxidativo, inflamações diversas das vias aéreas e redução da capacidade de defesa dos pulmões [25].

Figura 2 - Deposição de partículas com diferentes tamanhos no sistema respiratório. Partículas menores se depositam em maior quantidade na região pulmonar

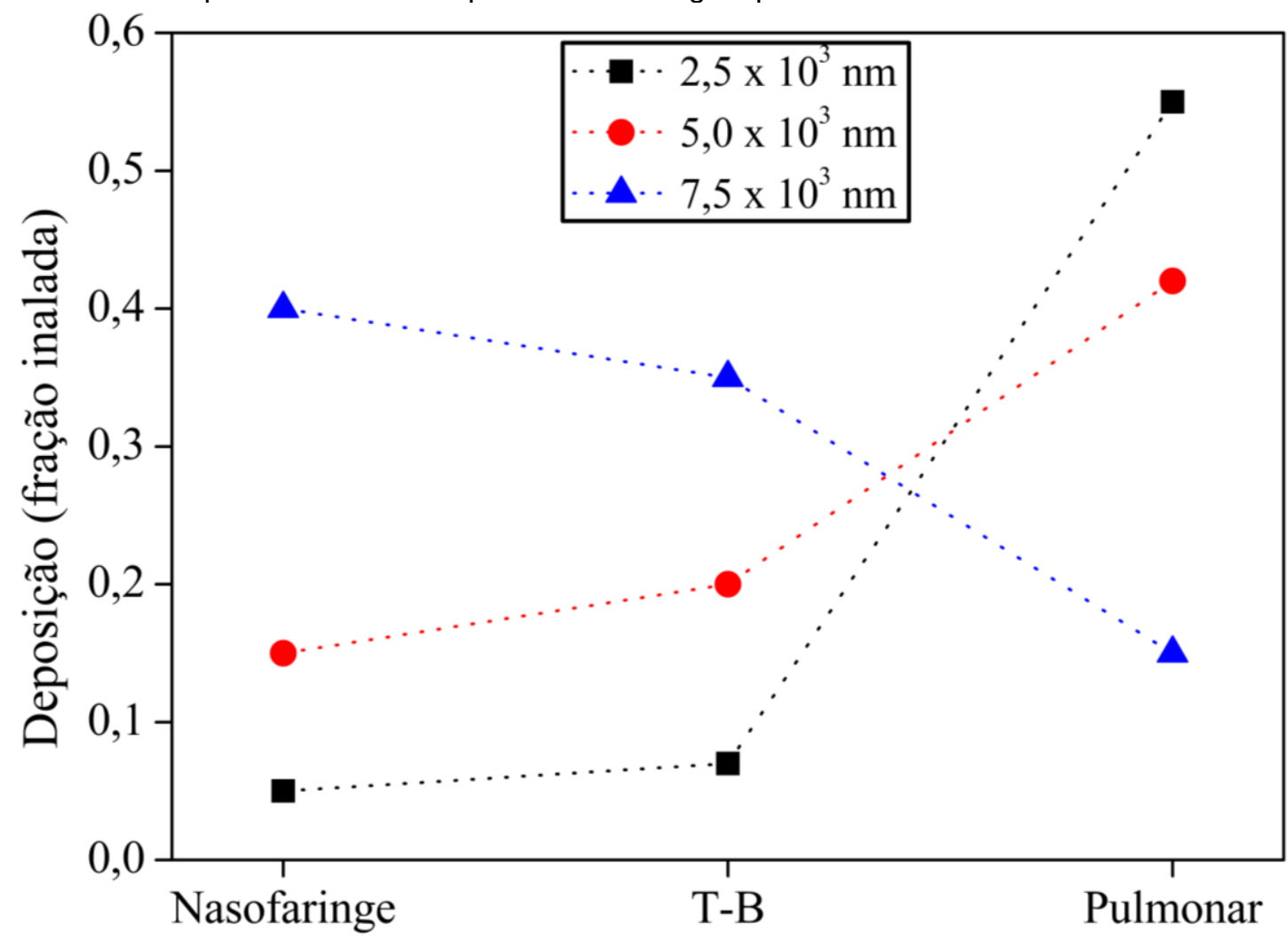

Região do Sistema Respiratório

Fonte: Modificada a partir de [26]

A nanotoxicologia pode ser definida como um ramo da ciência que tem como foco estudar os principais efeitos da toxicidade dos nanomateriais em organismos vivos [27]. Diversos fatores físico-químicos podem influenciar na toxicidade dos materiais, tais como: estrutura cristalina, morfologia, área superficial específica e carga superficial da partícula, tamanho médio dos cristalitos, solubilidade e atividade catalítica [28]. A presença ou ausência de uma casca e grupos ativos na superfície pode modificar a toxicidade dos nanomateriais 
[28]. O tamanho das nanopartículas (NPs) possibilita que elas atravessem às membranas celulares dos organismos vivos, ocasionando danos às células [29].

Estudos revelaram os efeitos químicos e biológicos de nanopartículas com diâmetros que variavam entre 14 e $95 \mathrm{~nm}$ [30]. Os autores constataram que as NPs com menor dimensão possuíam maior capacidade oxidativa que poderia ocasionar graves danos as células epiteliais alveolares. Nanoestruturas à base de carbono foram estudadas buscando entender o efeito da citotoxicidade em células cancerosas de ratos. Foi constatado que os diferentes tipos de nanomateriais utilizados possuíam morfologia distinta, além disso, a citotoxicidade foi avaliada com 3, 5, 7 e 10 dias [31]. Os autores verificaram que nanomateriais a base de grafeno apresentaram alta toxicidade $(52,24 \%)$, seguidos por nanotubos de carbono, materiais a base de fulereno e finalmente nanofios de carbono. Esse resultado confirma que a relação entre toxicidade e estrutura cristalina do material é um parâmetro de extrema relevância, mostrando a complexidade para desenvolver protocolos de segurança amplos para nanomateriais [31].

A dermatoxicidade pode ser entendida como os danos que substâncias químicas causam a pele dos seres vivos [29]. Essa interação pode originar diversas doenças, tais como: reações cutâneas, dermatite alérgica e fotossensibilização [32]. A pele se caracteriza como uma das principais rotas de contaminação aos nanomateriais, sendo necessário um melhor entendimento de seus mecanismos moleculares e potenciais riscos de exposição [33]. A citotoxicidade do grafeno na pele pode ser monitorada através do controle das atividades mitocondriais nas células [34]. Nessa obra, os autores descobriram que as nanoestruturas de grafeno compactadas provocam alta citotoxicidade nos fibroblastos, gerando mais espécies reativas de oxigênio (ROS). Entretanto, outros autores afirmam que até o momento, poucos estudos acerca da dermatoxicidade são publicadas pela comunidade científica, restringindo um melhor entendimento acerca deste importante tema [29].

Pesquisas que estudam o efeito da nanotoxicologia em seres vivos cresceram nas últimas décadas, entretanto, a quantidade de informações ainda é insuficiente para algumas conclusões. As lacunas existentes sobre esse tema dificultam determinar quais medidas preventivas devem ser utilizadas para evitar ou minimizar a exposição dos seres vivos no ambiente de trabalho. A área 
superficial das nanopartículas é uma propriedade importante para avaliar a toxicidade dos nanomateriais, como citado anteriormente, entretanto, o alto custo de aquisição e dificuldade de utilização em avaliações ambientais configuram um grande desafio para a engenharia de segurança moderna.

\section{PROCEDIMENTO DE SEGURANÇA EM LABORATÓRIOS}

A elaboração de um procedimento de segurança para laboratórios que manipulem nanotubos de carbono não é uma tarefa trivial, pois envolve diversos fatores, tais como: capacitação da equipe de trabalho, avaliação de risco e estrutura física do laboratório. O risco associado a essa atividade em laboratórios pode ser minimizado a partir da implementação de medidas preventivas. A seguir são dispostas algumas considerações importantes para o manuseio de nanotubos de carbono em laboratórios.

1. A detecção da contaminação por nanopartículas transportadas pelo ar ainda é um grande desafio para a engenharia moderna. Com base nisso, a adoção de um sistema de ventilação local, onde o pesquisador irá manusear nanomateriais, evitando que as nanopartículas fiquem aglomeradas no ar, se caracteriza como uma eficiente medida de prevenção, evitando a contaminação pelo sistema respiratório ou através da pele.

2. Utilização de um filtro tipo HEPA na saída do exaustor para retenção das nanopartículas conforme indicado pela National Institute for Occupational Safety and Health (NIOSH).

3. Criação de um guia claro e conciso informando as etapas necessária para criação dos nanotubos de carbono, levando em consideração o grau de risco quanto a contaminação (Baixo, Médio ou Alto). Tal medida preventiva, ajudaria ao pesquisador, entender a importância de se proteger durante a execução do trabalho.

4. Definir a quantidade máxima de nanomateriais manipulados durante o dia reduziria o risco de contaminação pelo sistema respiratório ou pela exposição à pele [35].

5. Manusear as matérias primas para fabricação dos nanotubos de carbono em uma capela fechada para evitar derramamentos e possíveis contaminações [36]. 
6. Realizar treinamentos periódicos informando aos pesquisadores os riscos que estão associados à manipulação dos nanomateriais para fabricação dos NTC.

7. Definir uma destinação adequada para os resíduos provenientes da fabricação de nanotubos de carbono, uma vez que esse material é tóxico para os seres humanos. O ensacamento específico para esse tipo de resíduo, seguido de um sepultamento em local apropriado seria uma possível solução.

8. Destinar uma balança de pesagem específica para manipulação dos nanomateriais que darão origem aos NTC, no intuito de evitar qualquer tipo de contaminação.

9. Utilização de um aspirador de pó com filtro HEPA para eliminação de pó solto.

10. Limpeza de superfícies utilizando lenços umedecidos ou aspirador com filtro HEPA conforme necessário ao longo do dia e pelo menos no final de cada turno de trabalho ${ }^{40}$.

11. Caso haja derramamento de líquidos contendo nanomateriais utilizar materiais absorventes e descartar os resíduos utilizando procedimentos de resíduos perigosos ${ }^{40}$.

12. Uma avaliação de risco adequada e detalhada deverá ser realizada semestralmente ou por necessidade específica, por um profissional legalmente habilitado, buscando garantir que os usuários estejam cientes de como proceder para mitigar o problema relacionado aos nanomateriais utilizados, não apenas para fabricação dos NTCs, mas a outras matérias primas utilizadas no laboratório [37].

13. Materiais à base de grafeno não devem ser descartados em pias ou ralos, além disso, em situações especiais, que utilizem líquidos de decantação contendo grafeno deverá ser contato empresas específicas de eliminação de resíduos químicos [38].

Outra forma de minimizar a possibilidade de contaminação em laboratórios é utilizando EPI's adequados. A figura 3 demonstra alguns tipos de equipamentos que devem ser utilizados para manusear nanomateriais a base de carbono. 
Figura 3 - Modelos de equipamentos de proteção individual indicados para manipular nanotubos de carbono.

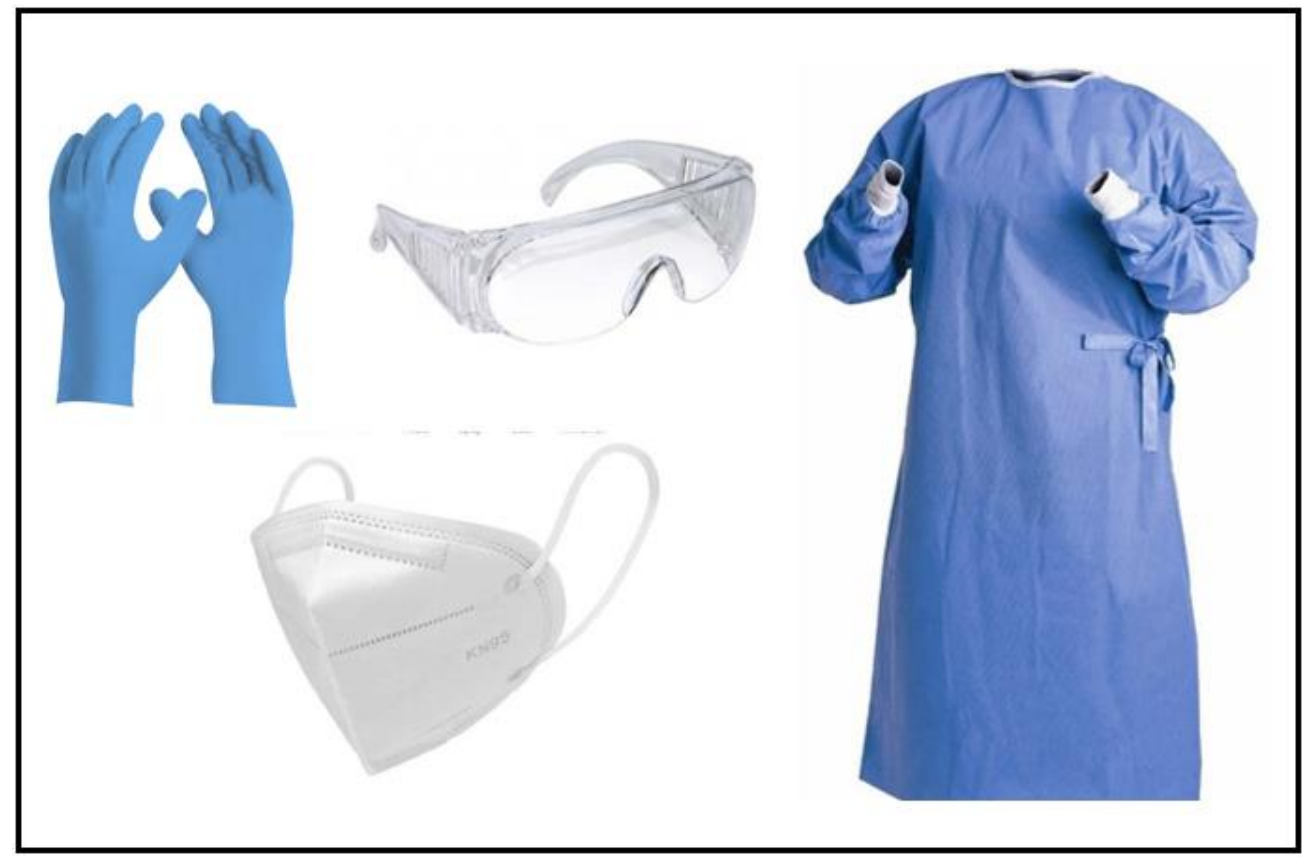

A figura 3 apresenta equipamentos de proteção individual indicados para manuseio de nanomateriais. É importante ressaltar que a utilização de EPI é requerida quando controles de engenharia e medidas administrativas não asseguram níveis de contaminação abaixo do limite aceitável. Devem ser utilizadas luvas nitrílicas ou a base de outro material quimicamente impermeável; as luvas devem ser inspecionadas antes do uso e substituídas assim que apresentarem indícios de desgaste [39]. O avental deve ser do tipo cirúrgico estéril impermeável, em busca de garantir a não contaminação através da pele. Os óculos de proteção devem ser do tipo incolor com ampla visão. É indicado utilizar respiradores faciais com filtro N100, N99 ou N95 pois garantem uma eficiência mínima de 99,97\%, 99\% e 95\%, respectivamente, para partículas sólidas [40].

Uma técnica que vem sendo amplamente utilizada na avaliação de risco à exposição aos nanomateriais é a de controle por faixas (control banding) [7]. 0 control banding é uma proposta de sistema de gestão e de estratégia de prevenção que auxilia gestores de pequenas e médias empresas na realização da avaliação dos riscos de forma qualitativa, sem a exigência de grande capacitação dos gestores [41]. Essa metodologia fornece resultados de avaliação com uma qualidade razoável, sem o envolvimento de especialistas [7]. Essa técnica possui quatro faixas que podem ser visualizadas na figura 4 . 
Figura 4 - Hierarquia tradicional de práticas para controle de exposição utilizada na técnica control banding.

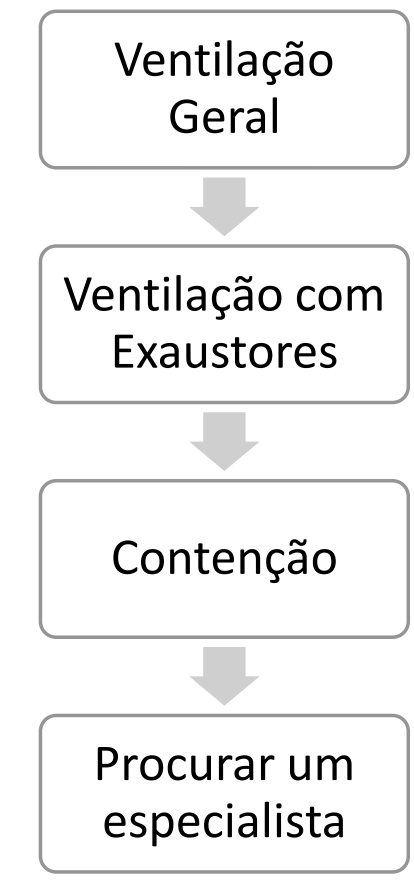

Fonte: Adaptado a partir de [42]

As faixas de controle devem ser usadas em conjunto com práticas de saúde e segurança do trabalho. Pela ordem hierárquica é indicado, por exemplo, que o ambiente de trabalho esteja devidamente ventilado de forma natural; caso essa medida não seja suficiente a próxima faixa deve ser adotada, ou seja, ventilação automatizada com utilização de exaustores deve ser adotada e assim sucessivamente, sempre seguindo a ordem hierárquica proposta pela técnica de controle por faixas.

\section{CONSIDERAÇÕES FINAIS}

O conhecimento acerca da manipulação, descarte e danos causados à saúde devido a interação entre os nanomateriais, inclusive os NTC, e os seres humanos ainda são um grande paradigma. Entretanto, o avanço na saúde e segurança do trabalho vem propiciando uma melhoria nas avaliações de risco, devido a inúmeros trabalhos publicados na literatura e pesquisas que envolvem segurança no trabalho. Já existem equipamentos capazes de mensurar a exposição ocupacional de um ser humano, levando em consideração a concentração em massa das nanopartículas no ar expirado. Porém, esse dado 
não é suficiente para avaliar o risco. Ainda há necessidade de desenvolver equipamentos não individuais que coletam amostras de ar e possibilitam verificar a distribuição das nanopartículas pelo tamanho. Entretanto, é sabido afirmar que a exposição a nanomateriais, dentre esses os nanotubos de carbono, podem ser absorvidos pelo sistema respiratório e através do contato com a pele, podendo causar danos irreparáveis a saúde dos trabalhadores.

Com base nisso, medidas de prevenção que busquem minimizar os riscos de contaminação e protocolos claros e concisos para manipulação de nanomateriais em laboratórios devem ser elaborados e adotados. Outra via, os nanomateriais são uma classe ampla e que continua se expandido, sendo necessário o estudo por classes de materiais, como foi feito nessa pesquisa, dando ênfase aos nanotubos de carbono, que são materiais com características peculiares. Os laboratórios que manipulam esse tipo de tecnologia devem ser dotados de instalações específicas e possuir procedimentos operacionais que busquem controlar a exposição e a contaminação aerotransportada. A generalização quando se trata de nanomateriais é muita perigosa, uma vez que, cada nanomaterial é desenvolvido para atender necessidades específicas, possuindo riscos diferentes ao corpo humano, devendo esse tratamento amplo ser evitado.

A compatibilização da cadeia comercial e a segurança do trabalho deve ser considerada para nanomateriais, ou seja, o diálogo entre fabricante, fornecedor, usuário final e órgãos e profissionais da segurança do trabalho são de suma importância para o desenvolvimento da nanotecnologia com segurança. É importante ressaltar que as considerações descritas nessa pesquisa utilizaram conhecimentos disponíveis até o ano vigente, à saber, 2021, e em futuras avaliações os parâmetros podem mudar significamente, pois a nanotecnologia e seus produtos estão em uma crescente logarítmica. 


\section{REFERÊNCIAS}

[1] Khan I, Saeed K, Khan I. Nanoparticles: Properties, applications and toxicities. Arabian journal of chemistry. 2019;12(7):908-931.

[2] Duhan J. S., et al. Nanotechnology: The new perspective in precision agriculture. Biotechnology Reports. 2017;15:11-23.

[3] Wang K. et al. Síntese de nanotubos de carbono hidrofóbicos / filmes compostos de óxido de grafeno reduzido por irradiação com luz flash. Fronteiras da Ciência Química e Engenharia. 2018;12(3):376-382.

[4] Groso A. Engineered nanomaterials: toward effective safety management in research laboratories. Journal of nanobiotechnology. 2016;14(1):1-17.

[5] Turini C. A. Avaliação in vivo da biodistribuição e efeitos tóxicos de nanotubos de carbono de paredes múltiplas em camundongos [Monografia]. Brasil: Universidade Federal de Uberlândia; 2018. Bacharel em Biomedicina.

[6] Silva F. Risk assessment and control in engineered nanoparticles occupational exposure. Occupational Safety and Hygiene. 2013;:197-202.

[7] Hashimoto, H. et al. Evaluation of the control banding method-comparison with measurement-based comprehensive risk assessment. Journal of occupational health. 2007;49(6):482-492.

[8] Mohan V. B. Handling and risk mitigation of nanoscale graphene and related materials: some considerations and recommendations. C-Journal of Carbon Research. 2019;5(3):26.

[9] Trojanowicz, M. Analytical applications of carbon nanotubes: a review. TrAC trends in analytical chemistry. 2006;25(5):480-489.

[10] Chavan, R. et al. A review: Carbon nanotubes. Int. J. Pharm. Sci. Rev. Res. 2012;13(1):124-134.

[11] Saifuddin N., Raziah A. Z, Junizah A. R. Carbon nanotubes: a review on structure and their interaction with proteins. Journal of Chemistry. 2013.

[12] Pujals D. C, et al. XPS de nanoestructuras de carbono obtenidas por descarga de arco de electrodos de grafito sumergidos en água. Nucleus. 2018;(64):15-18.

[13] Moreira J. V. S, Almeida P. L. R, Zanin H. G. Caracterização de nanotubos de carbono de paredes múltiplas autossustentáveis como dispositivos supercapacitores. Revista Univap. 2017;22(40):910.

[14] Cunha THR, et al. High-yield synthesis of bundles of double-and triple-walled carbon nanotubes on aluminum flakes. Carbon. 2018;133:53-61. 
[15] Liné C, Larue C, Flahaut E. Carbon nanotubes: Impacts and behaviour in the terrestrial ecosystem-A review. Carbon. 2017;123:767-785.

[16] Ma-Hock L, et al. Inhalation toxicity of multiwall carbon nanotubes in rats exposed for 3 months. Toxicological Sciences. 2009;112(2):468-481.

[17] Romero JGV, et al. Síntese de nanotubos de carbono de parede simples por sublimação de grafite em atmosfera de hélio. Química Nova. 2002;5(1).

[18] Wang L, et al. Dispersion of single-walled carbon nanotubes by a natural lung surfactant for pulmonary in vitro and in vivo toxicity studies. Particle and fibre toxicology. 2010;7(1):1-10.

[19] National Institute for Occupational Safety and Health (NIOSH). Nanomaterial production and downstream handling processes. (2013). Disponível em: https://www.cdc.gov/niosh/docs/2014-102/. Acesso em: 17 de novembro de 2020.

[20] Warheit DB, et al. Comparative pulmonary toxicity assessment of single-wall carbon nanotubes in rats. Toxicological sciences. 2004;77(1):117-125.

[21] Jesus KRE, Castro VLSS. Nanotoxicologia aliada à avaliação de riscos ambientais: uma estratégia para avaliações de segurança das nanotecnologias no Brasil. Embrapa Meio Ambiente; 2013; São Carlos; 2013. 525-527.

[22] Freitas AM, et al. Characterization of PM10 and PM2. 5 and size distribution of chloride, nitrate and sulphate in urban and rural atmospheres of Londrina. Química Nova. 2009;32(7):1750-1754.

[23] Bagatin E, et al. Granulomatous diseases of occupational etiology. J Bras Pneumol. 2006;32:69-84.

[24] Dias AG, et al. Riscos Ocupacionais em Atividade de Coleta de Resíduos Sólidos. E\&S Engineering and Science. 2015;3(1):3-17.

[25] Godish, T. Air Quality. Boca Raton: CRC Press LLC, 2004.

[26] Holgate, S. T. Air pollution and health. San Diego: Academic Press, 1999.

[27] Zhao Y, et al. Nanotoxicology: Toxicological and biological activities of nanomaterials. Nanoscience and nanotechnologies. 2012;1-53.

[28] Sukhanova A, et al. Dependence of nanoparticle toxicity on their physical and chemical properties. Nanoscale research letters. 2018;13(1):44.

[29] Madannejad R, et al. Toxicity of carbon-based nanomaterials: Reviewing recent reports in medical and biological systems. Chemico-biological interactions. 2019;307:206-222.

[30] Koike E, Kobayashi T. Chemical and biological oxidative effects of carbon black nanoparticles. Chemosphere. 2006;65(6):946-951. 
[31] Srikanth M, et al. Effects of morphology, concentration and contact duration of carbon-based nanoparticles on cytotoxicity of 1929 cells. ASME International. 2015.

[32] Ali N, Oehme FW. A literature review of dermatotoxicity. Veterinary and human toxicology. 1992;34(5):428-437.

[33] Ding L, et al. Molecular characterization of the cytotoxic mechanism of multiwall carbon nanotubes and nano-onions on human skin fibroblast. Nano letters. 2005;5(12):2448-2464.

[34] Liao $\mathrm{KH}$, et al. Cytotoxicity of graphene oxide and graphene in human erythrocytes and skin fibroblasts. ACS applied materials \& interfaces. $2011 ; 3(7): 2607-2615$.

[35] Bussy C, Ali-Boucetta H, Kostarelos K. Safety considerations for graphene: lessons learnt from carbon nanotubes. Accounts of chemical research. 2013;46(3):692-701.

[36] Laux P, et al. Nanomaterials: certain aspects of application, risk assessment and risk communication. Archives of toxicology. 2018;92(1):121-141.

[37] Seabra $A B$, et al. Nanotoxicity of graphene and graphene oxide. Chemical research in toxicology. 2014;27(2):159-168.

[38] Kong B, et al. Experimental considerations on the cytotoxicity of nanoparticles. Nanomedicine. 2011;6(5):929-941.

[39] National Institute for Occupational Safety and Health - NIOSH. Workplace design solutions: Protecting Workers during the Handling of Nanomaterials, (2018). Disponível em https://www.cdc.gov/niosh/docs/2018-121/pdfs/2018121.pdf. Acesso em 12 de abril de 2021.

[40] National Institute for Occupational Safety and Health - NIOSH. 42 CFR Part 84 Respiratory Protective Devices, (1997). Disponível em https://www.cdc.gov/niosh/npptl/topics/respirators/pt84abs2.html. Acesso em 12 de abril de 2021.

[41] Fundacentro. Disponível em https://www.gov.br/fundacentro/ptbr/assuntos/noticias/noticias/2018/5/especialista-da-oms-elogia-o-trabalho-dafundacentro. Acesso em 12 de abril de 2021.

[42] Zalk, D. M., Heussen, G. H. Banding the world together; the global growth of control banding and qualitative occupational risk management. Safety and health at work. $2011 ; 2(4): 375-379$. 